\title{
Research on detection method of load in wave compensation system based on MTi
}

\author{
Jiandong Zhao,Limin Tao,Wei Lv \\ National University of Defense Technology, Changsha, China \\ *452288680@qq.com
}

Keywords: MTi; Wave compensation system; attitude angle; data acquisition

\begin{abstract}
Aiming at the demand of high precision real-time acquisition of load attitude in wave compensation system, a method based on MTi to detect load attitude is proposed in this paper. Based on the data acquisition card and $\mathrm{C}++$ Visual language, the on-line acquisition and calculation of the Euler angle of wave compensation system is realized, and the signal acquisition hardware system and software system are designed. The experimental results show that the detection method can detect the attitude of the load in real time and the detection method provides a reliable parameter for the trajectory planning and motion control of the load.
\end{abstract}

\section{Introduction}

Muti-DOF heave compensation crane can compensate the relative movement of two ships to eliminate the influence of replenishment operation at sea. Suspended cable driven parallel mechanism (SCDPM) is an important part of the heave compensation crane [1]. It is a new kind of wave compensation equipment in offshore cargo handling, which compensates the wave-induced ship 6-dof motion. In the wave compensation system, the position and attitude of the load need to be controlled. In order to achieve the accurate control of the load position, the position and attitude of the load are detected and fed back to the control system in real time. The load position detection includes rolling, pitching and yawing three Euler angles [2]. The accuracy and real-time of the load attitude detection parameters are directly related to the accuracy of the whole wave compensation system. Therefore, the attitude detection is an important task.

The existing attitude detection methods include GPS, vision, inertia detection and so on. The disadvantage of GPS detection is the need to install the receiver base, and the need to carry out the experiment outdoors, so that the inconvenience to the experiment [3]. The disadvantage of the visual inspection system is that the mark of the visual detection system is complex and various, and the solving speed is relatively slow. In addition, the visual detection system is affected by the environment. In the rain, fog and other poor visibility conditions, the detection precision is greatly affected by the environment [4]. But the inertial device MTi can get the attitude information quickly and accurately, and the solution is simple and real-time is really satisfying [5], which can provide reliable parameters for the wave compensation control system.

\section{The Principle of MTi}

MTi which is produced in Holland Xsens company is a micro product. It can accurately measure the attitude and heading and inertial parameters. Appearance is shown in figure 1. MTi uses its internal enhanced three axis gyro to quickly track the spatial pose of the object measured and simultaneously measures the 3 axial acceleration and the ground magnetic field to provide compensation for the 3 axis speed. Through its internal integration of the sensor fusion algorithm, MTi puts the attitude information into the system through the USB serial communication bus interface in real time. It has the advantages of small size, light weight, low power consumption and low cost. It is mainly used in the field of robot attitude control, unmanned aerial vehicle navigation, ship attitude detection and control, tunnel excavation and drilling, etc. MTi can be easily integrated into other systems, and re-development, which has a wide range of applications. The typical direction error data: Angular 
resolution is 0.05 degrees; Static accuracy(roll/pitch) is less than 0.5 degrees; Dynamic accuracy is less than 1.0 degrees.

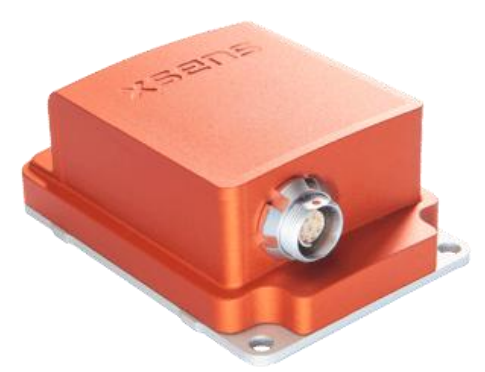

Figure 1. The Appearance of MTi

The MTi is calculated by the coordinate system of the sensor(xyz) and the coordinate system of the magnetic field of the earth(XYZ). The coordinate system is shown in Figure 2.

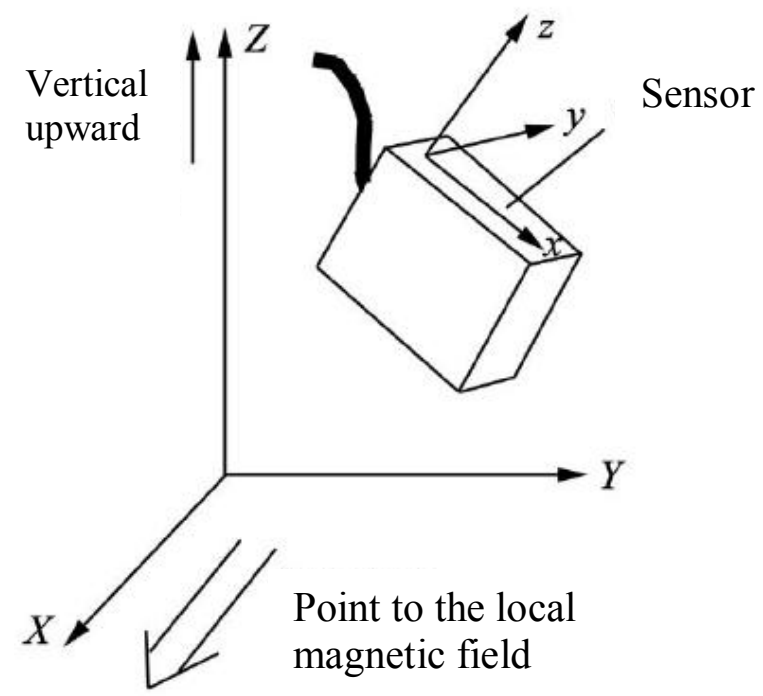

Figure 2. The sensor coordinate system and the earth's magnetic field coordinate system

MTi is derived by calculating the 3 angles between the sensor coordinate system (XYZ) and the local Descartes right hand coordinate system (XYZ). Attitude angle can be represented by different parameters, such as the quaternion, Euler angle and direction cosine matrix.

\section{Mathematical description of three dimensional motion attitude}

\subsection{Description of the quaternion}

The quaternion vector can be considered as a description of the rotation angle $\alpha$ of a unit vector $n$. The quaternion vector is a four element vector $q=\left(q_{0}, q_{1}, q_{2}, q_{3}\right)$, which can be use to represent the attitude. Practice has proved that the quaternion can be given a very good numerical solution. As we know, the Euler angles of the three rotations can be represented by angular positions. And rotation can be expressed by the quaternion, so Euler angle is bound to determine the relationship between the quaternion. In the transformation of the two coordinate systems, the quaternion and the Euler angles are represented by the coordinate transformation:

$$
\left(\begin{array}{ccc}
\cos \theta \cos \psi & \cos \theta \sin \psi & -\sin \theta \\
\sin \varphi \sin \theta \cos \psi-\cos \varphi \sin \psi & \sin \varphi \sin \theta \sin \psi+\cos \varphi \cos \psi & \sin \varphi \cos \theta \\
\cos \varphi \sin \theta \cos \psi+\sin \varphi \sin \psi & \cos \varphi \sin \theta \sin \psi-\sin \varphi \cos \psi & \cos \varphi \cos \theta
\end{array}\right)
$$




$$
\left(\begin{array}{ccc}
q_{0}^{2}+q_{1}^{2}-q_{2}^{2}-q_{3}^{2} & 2 q_{0} q_{3}+2 q_{1} q_{2} & -2 q_{0} q_{2}+2 q_{1} q_{3} \\
-2 q_{0} q_{3}+2 q_{1} q_{2} & q_{0}^{2}-q_{1}^{2}+q_{2}^{2}-q_{3}^{2} & 2 q_{0} q_{1}+2 q_{2} q_{3} \\
2 q_{0} q_{2}+2 q_{1} q_{3} & -2 q_{0} q_{1}+2 q_{2} q_{3} & q_{0}^{2}-q_{1}^{2}-q_{2}^{2}+q_{3}^{2}
\end{array}\right)
$$

Achieve attitude angle:

$$
\begin{aligned}
& \sin \theta=2 q_{0} q_{2}-2 q_{1} q_{3} \\
& \tan \psi=\frac{2 q_{0} q_{3}+2 q_{1} q_{2}}{q_{0}^{2}+q_{1}^{2}-q_{2}^{2}-q_{3}^{2}} \\
& \tan \varphi=\frac{2 q_{0} q_{1}+2 q_{2} q_{3}}{q_{0}^{2}-q_{1}^{2}-q_{2}^{2}+q_{3}^{2}}
\end{aligned}
$$

The above three values can be obtained by the Anti sine and Anti tangent and the attitude angle can be obtained. But it should be noted that the arctangent in four quadrant inverse tangent function.

\subsection{Description of the direction cosine matrix}

The direction cosine matrix is often used to describe the relative attitude between the two coordinate systems in the inertial navigation system analysis. The rotation matrix can be considered as a description of the rotation of the unit vector in the sensor coordinate system with respect to the earth's magnetic field coordinate system. For a rigid body coordinate system of a position, using direction cosine can be very convenient to coordinate transformation. The coordinate transformation of a vector in a coordinate system is represented by the coordinate of the other coordinate system. The advantage of direction cosine matrix is that it is easy to make vector conversion between two coordinate systems.

\subsection{Description of Euler angle}

Euler angle is the angle of the space generated by $\mathrm{X}, \mathrm{Y}$, and $\mathrm{Z}$ axis rotation of the target in the field of the geomagnetic field coordinate system. Euler angle includes roll $\phi, \operatorname{pitch} \theta$ and yaw $\varphi$. The Euler angle defined by MTi is in the range of: $\phi=$ around the $\mathrm{X}$ axis rotation, rotation angle $\left[-180^{\circ} \sim 180^{\circ}\right]$; $\theta=$ around the $\mathrm{Y}$ axis rotation, rotation angle $\left[-90^{\circ} \sim 90^{\circ}\right] ; \varphi=$ around the $\mathrm{Z}$ axis rotation, rotation angle $\left[-180^{\circ} \sim 180^{\circ}\right]$; Because of the mathematical character of the definition of Euler angles, when the $\mathrm{X}$ axis in the coordinate system of the sensor is pointed up or down in the magnetic field coordinate system, that is, when the $\mathrm{Y}$ axis is rotated to $\pm 90^{\circ}$, there will be a Singularity Phenomenon that the angle will reach $180^{\circ}$ in short time. In the vicinity of $\theta= \pm 90^{\circ}$, Minor changes in the angle $\theta$ can also cause large fluctuations in the angle $\varphi$ and $\phi$. This shows that the system is weak in the vicinity of $\theta= \pm 90^{\circ}$. In practical application, it should be avoided as far as possible. Just because of the existence of this singularity, the direct output of MTi Euler angle is a potential problem. The benefit of Euler angles is intuitive. So the relationship between the rotation matrix represented by the quaternion and the rotation matrix represented by the Euler angle should be derived, then the Euler angle will be calculated.

\section{Working state of MTi and the binary communication protocol of MTi}

\subsection{Working state of MTi}

The MTi has two states, i.e. Config State and Measurement State. In the Config State various settings can be read and written, for example, the sampling interval, the output baud rate setting, output mode etc. In the Measurement State the device will output its data message which contains data dependent on the current configuration. There are two different ways to enter the Config State or the Measurement State. At power-up the device starts the WakeUp procedure, if no action is taken it will then enter Measurement State by default, using its latest stored configuration. Prior to entering the Measurement State, the Configuration and eMTS(extended Motion Tracker Specification) messages are always sent to the host. Configuration data is the configuration that is read from the internal non-volatile memory and will be used in the Measurement State. The data in the Configuration message can always be used to determine the output mode and settings. It is also possible to enter the 
Config State at power-up, see WakeUp message description in the MT Low-Level Communication Document. Another way to enter the Config State or Measurement State is to use the GoToConfig or GoToMeasurement messages. The eMTS data is required to be able to later process the data by Xsens software to calculate calibrated inertial data values as well as estimating orientation etc. The figure 3 shows the working state of MTi [6][7].

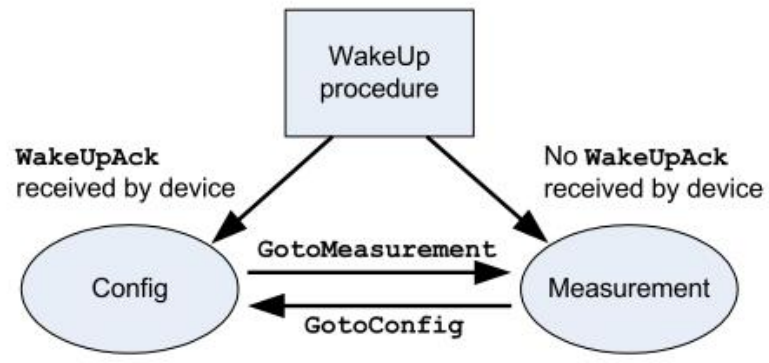

Figure 3. The working state of MTi

\subsection{The binary communication protocol of MTi}

The communication with the MTi is done by messages which are built according to a standard structure. The standard MT message can contain zero to 254 bytes of data and the total length is five to 259 bytes. An MT message contains the following fields:

\begin{tabular}{|l|l|l|l|l|l|}
\hline PRE & BID & MID & LEN & DATA & CS \\
\hline
\end{tabular}

Field, field width and description of MT message are shown as Table 1.

\begin{tabular}{|c|c|c|}
\hline Field & Field width & Description \\
\hline PRE & 1 byte & Preamble, indicator of start of packet $\rightarrow 250$ (0xFA) \\
\hline BID & 1 byte & Bus identifier / address $\rightarrow 255$ (0xFF) \\
\hline MID & 1 byte & Message identifier \\
\hline LEN & 1 byte & $\begin{array}{l}\text { Value equals number of bytes in DATA field } \\
\text { Maximum value is } 254 \text { (0xFE). Value } 255 \text { (0xFF) is reserved. }\end{array}$ \\
\hline DATA & $0-254$ bytes & Data bytes (optional) \\
\hline CS & 1 byte & Checksum of message \\
\hline
\end{tabular}

Table 1. Field, field width and description of MT message

\section{Attitude detection system}

The host computer directly communicate with MTi through RS232 to set the output mode and output settings of MTi in this system. Taking into account the re-development of the program, a modular design ideas is used by the program. The procedure is mainly divided into three part: reading data, processing data and display data. The direction of the output mode is quaternion, and the current roll angle, pitch angle and yaw angle which are the 3D pose of the load are calculated in real time.

\subsection{Hardware design}

MTi is connected with the upper computer by RS232. At the same time, the external power supply for MTi power supply. MTi is fixed to the load. The corresponding Euler angles are obtained by calculating the values of the quaternion required for the acquisition then send data to the control system. Signal acquisition process is shown in Figure 4. Figure 5 provides loads along $X$ axis for the rolling load, along $\mathrm{Y}$ axis for pitching, along $\mathrm{Z}$ axis for yaw.

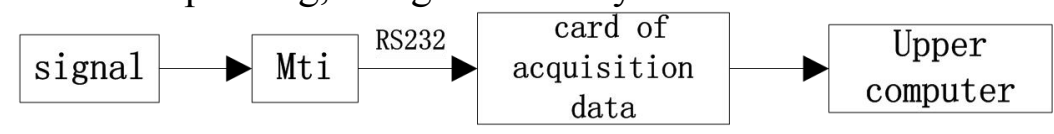

Figure 4. Signal acquisition process 


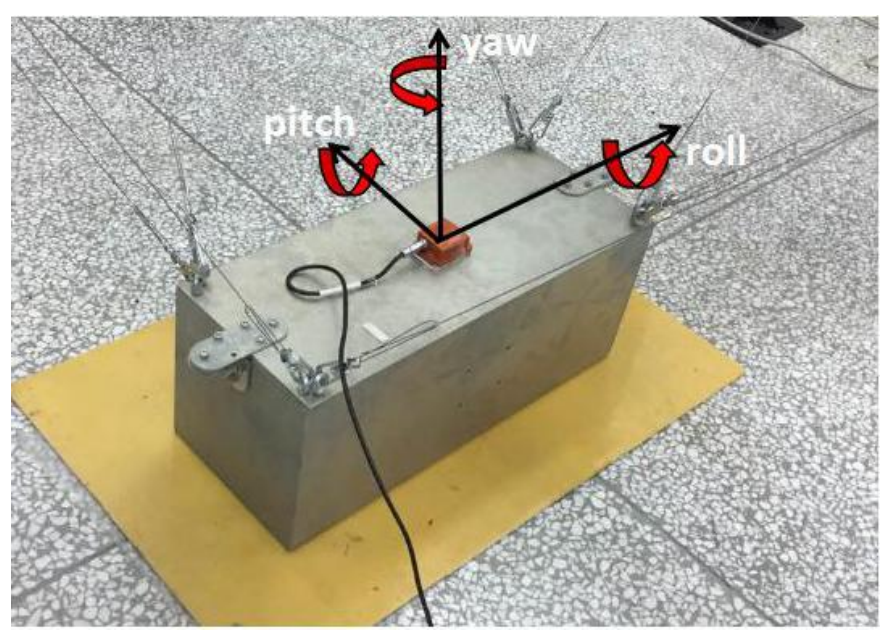

Figure 5. Physical map of load detection system

\subsection{Flow of Software control}

The VS2010 application program running on IPC is shown in figure 6. The application task mainly includes the initialization of wave compensation control system, the setting of control parameters, the online collection of MTi data, the online processing of MTi data, and the real-time display of the data.

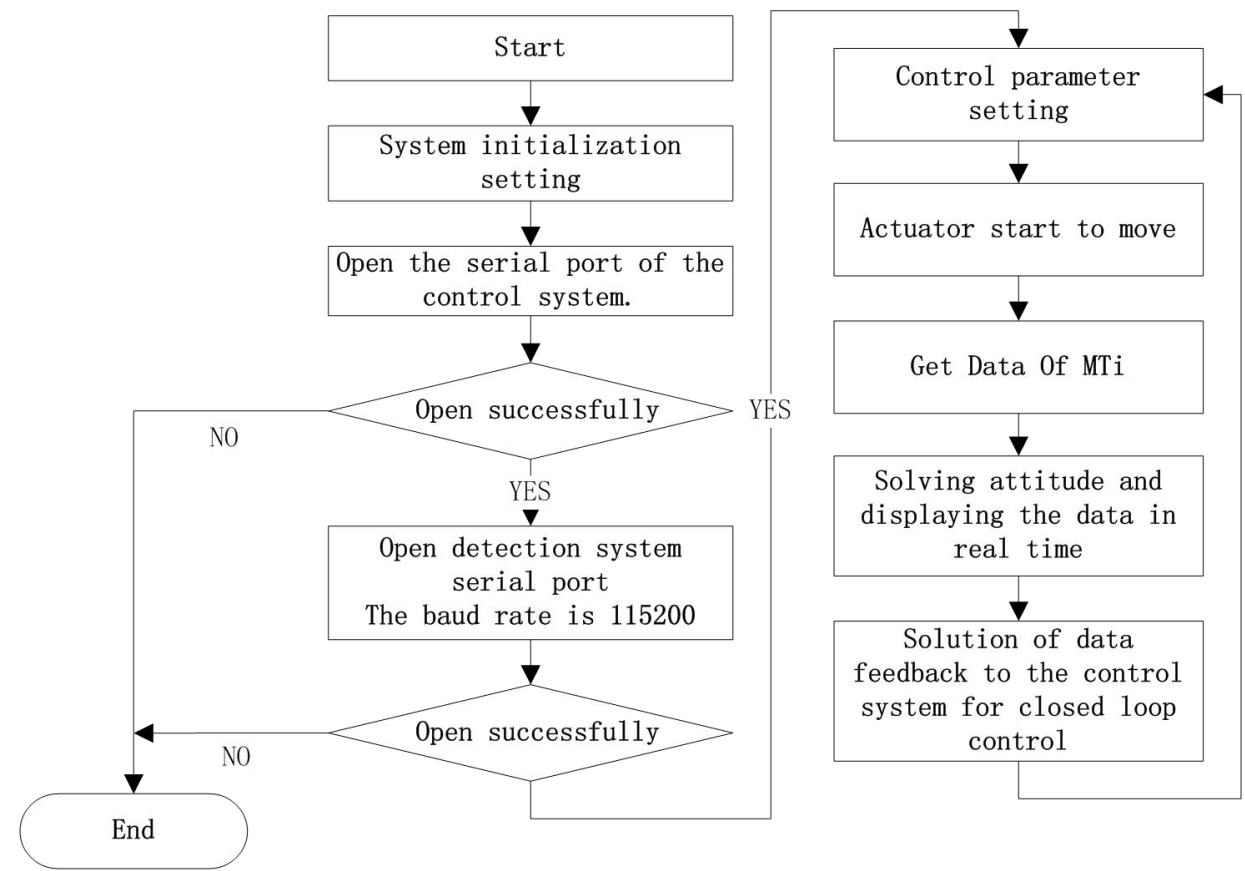

Figure 6. Application flow chart

\subsection{Experimental data processing and analysis}

In this system, $\mathrm{C}++$ Visual software is used for real time data acquisition, data processing and drawing curves. MTi is installed in the center of the load on the surface, and makes the MTi sensor coordinate system $\mathrm{X}$ axis and $\mathrm{Y}$ axis parallel to the $\mathrm{X}$ axis and the $\mathrm{Y}$ axis of the load coordinate system. The load is rotated around the three axis of the load in the eight wire-driven wave compensation system. Attitude angle detected by the MTi and the setting attitude angle of the wave compensation system are compared in real time.

First of all pitching experiment along the $\mathrm{Y}$ axis is tested. In $\mathrm{C}++$ Visual development $\mathrm{A}$ sine wave input signal with an amplitude of 10 degrees is set: load pitching $=10 \times \sin (t+0.628)$. The load is increased under the control of the rope, and then the load carries on the pitch movement around the $\mathrm{Y}$ axis. The attitude angle of the detecting system is displayed in real time through the TeeChart drawing plug-in in $\mathrm{C}++$ Visual, and the contrast of the set signal is formed at the same time, and the error curve is made. The experimental results are shown in figure 7. 

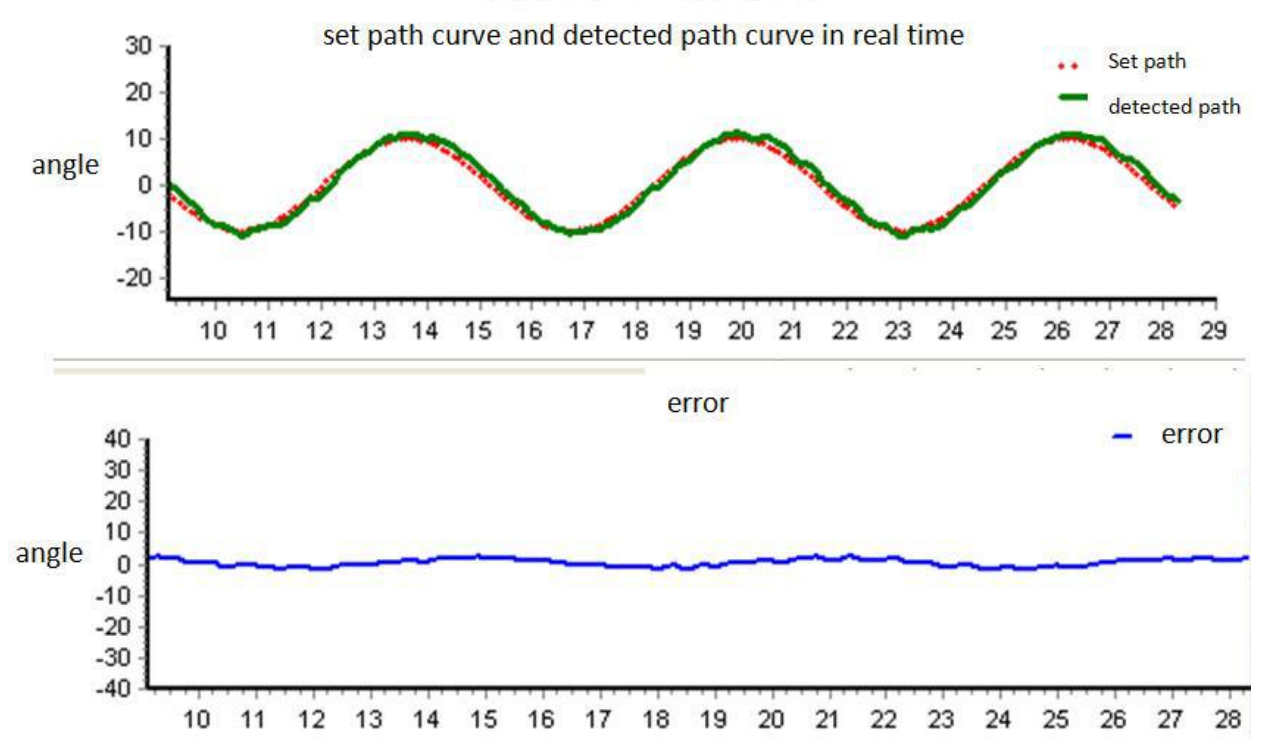

Figure 7. Curve of pitch and curve of error change with time

In the same method rolling test and yaw test of load is worked. Experimental results are shown in Figure 8 and Figure 9.
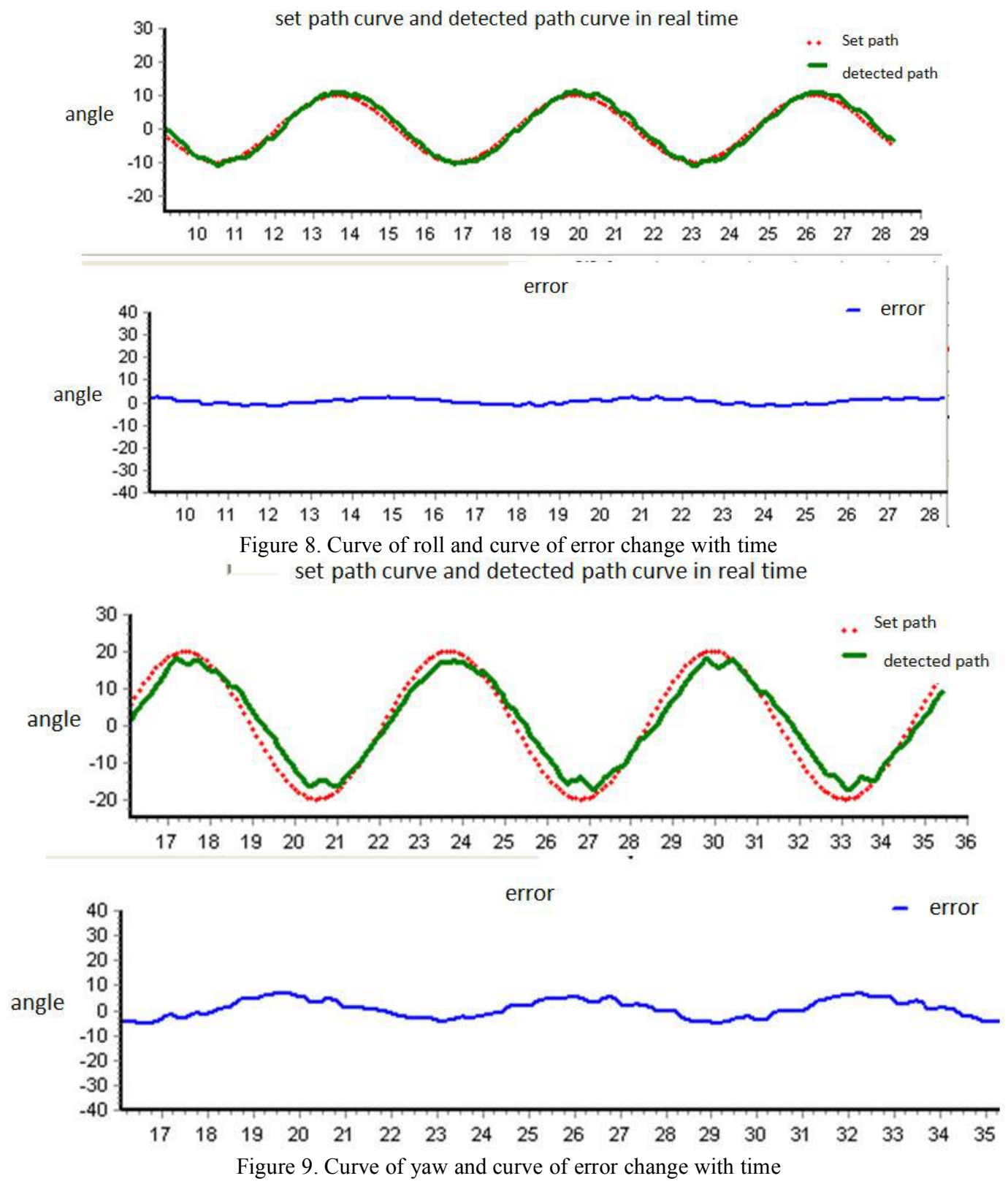
After many experiments, it is found that the pitch detection curve of Figure 7 is very close to the set curve. The experiment verifies the reliability of the detection system, and it also verifies the accuracy of the wave compensation control platform in the direction of rotation of the $\mathrm{Y}$ axis. Fig. 8 shows that there is a certain delay in the detection curve compared with the preset curve, which is caused by the wave compensation control system in the direction of the roll. In the Fig. 9 the curves of yaw also appeared in the delay phenomena to a certain extent, and on the crest of the wave trough, curve is not smooth, indicating that the control system in the yaw direction control is not stable. It should be noted that, due to the internal integration of the system, it is absolutely not to be placed in a strong magnetic field. But the control platform is an iron platform, and the motor is more, to a certain extent, it also affects the calibration of the magnetometer, resulting in the deviation of the yaw error. The next will do some experiments in the swing experiment improving. matrix represented by the Euler angle should be derived, then the Euler angle will be calculated.

\section{Conclusions}

In this paper, the load attitude of wave compensation system is detected based on the Mti sensor. The theoretical basis of MTi attitude sensor and its binary communication protocol are introduced in this paper. The software and hardware of attitude detection system are designed. The MTi test data is collected in real-time by $\mathrm{C}++\mathrm{Visual}$ programming and used in the control system. The experimental results show that the data acquisition system can accurately and timely collect the attitude parameters of wave compensation system load, and provide reliable parameters for accurate control of wave compensation system.

\section{References}

[1] Yongpan Hu, Limin Tao, Wei Lv. Anti-pendulation analysis of parallel wave compensation systems[J]. Proceedings of the Institution of Mechanical Engineers, Part M: Journal of Engineering for the Maritime Environment. 2014.

[2]Wei Lv, Limin Tao, Yongpan Hu. A novel control method for active heave compensation system $[\mathrm{C}]$. International Conference on Electronics, Communication and Control, Zhoushan, China, 2012.

[3] Xia Xianfeng, Wang Yuan, Zhang Hua, et al.GPS real-time attitude determination system based on MEMS inertia devices[J]. Transducer and Microsystem Technologies, 2010, 29(8): 141-144.(in Chinese)

[4] Lee J, Nam G, Lee B, et al. Measurement of 3D Spreader Position Information using the CCD Cameras and a Laser Distance Measuring Unit[J]. International Journal of Navigatioin and Port Research. 2004, 28(4): 323-331.

[5] John E Iwell. Progress on Micromechanical Inertial Instruments, AIA-912765-cp, 1482 1485

[6] MTi and MTx User Manual and technical Document Revision G. the Nertherland: Xsens, 2006

[7] MT Low Level Communication protocol Documentation Revision J. the Nertherland: Xsens,2008 